\title{
Wiederholbarkeit von Messungen und individueller Messfehler bei craniometrischen Untersuchungen an Apodemus
}

\author{
Hans M. STEINER \& Jan RACZYŃSKI
}

\begin{abstract}
Steiner H. M. \& Raczyński J., 1976: Wiederholbarkeit von Messungen und individueller Meßfehler bei craniometrischen Untersuchungen an Apodemus. Acta theriol., 21, 37: 535-541 [Mit 3 Tabellen].

Fourteen skulls of Apodemus flavicollis (M elchior, 1834) were measured in unbiased sequence by two experimenters. Each of the 8 measurements, also unbiased, was repeated three times. With the aid of an analysis of variance for each of the experimenters, repeatability and relative increase of accuracy from one to repeated measurements was computed. As expected, condylobasal-length and zygomatic breadth are the most accurate measurements of skull, but rostral depth, maxillary tooth-row length, length of diastema and length of ossa nasalia are accurate too. The results concerning depth of braincase and length of foramina incisiva are not satisfactory. It is only recommended to repeat measurements of those skulls which are very important. The minimum and maximum values in series should be tested again.

fInst. f. Experimentalle Zool. u. Vergl. Anat. u. Physiol. der Universität für Bodenkultur, Wien XVIII/110, Gregor Mendel-Str. 33, Austria; Mfammals Res. Inst., Polish Acad. Sci., 17-230 Białowieża, Poland]
\end{abstract}

\section{PROBLEMSTELLUNG}

Unterschiede zwischen Zwillingsarten sind oft zunächst vorwiegend biometrisch zu erfassen; dies gilt auch für die drei Angehörigen des Subgenus Sylvaemus in Europa (H a itlinger \& Ruprecht, 1967; $\mathrm{S}$ teiner, 1968; $\mathrm{N}$ i et ha m mer, 1969). In diesem Falle ist beim allgemein geringen Abstand der spezifischen Mittelwerte von für die Determination wesentlichen Maßen, die Meßgenauigkeit ein wesentlicher Faktor. Bei Verwendung von Meßdaten aus der Literatur ist man mit der Einschätzung des individuellen Meßfehlers konfrontiert. Deshalb führten wir die Fragen der Wiederholbarkeit von Maßen und des individuellen Meßfehlers der beiden Autoren einer experimentellen Prüfung zu.

\section{MATERIAL UND METHODE}

Aus einer Serie von in den Monaten April, Mai und Juli 1963 von Frau Dr. F'. Spitzenberger am Tulbinger Kogel (Wienerwald, N. Ö.) gesammelten Gelbhalsmäusen, Apodemus flavicollis (M elchior, 1834) wählten wir alle 14 intakten Schädel 
der Altersgruppe 4 und 5 (nach $\mathrm{S}$ te in er, 1968) für den Versuch. Wir einigten uns auf 8 Schädelmaße und verglichen vor dem Versuch unsere Meßtechniken grob. Wesentliche Unterschiede schienen sich hiebei nicht zu ergeben, Vergleichsmessungen führten wir nicht durch.

Ein Versuchsleiter überreichte in zufälliger Reihenfolge jedem der beiden Autoren einen Schädel, der dann einmal vermessen wurde. Der Versuchsleiter wiederholte die diktierten $\mathrm{Maße}$ und schrieb sie dann in eine Liste; Hörfehler sind also auszuschließen. Um grobe Ablesefehler von mehreren Millimetern auszuschalten, wurden wir in solchen Fällen gebeten, ein solches $\mathrm{Ma} \beta$ zu wiederholen. Uberraschenderweise waren innerhalb der 672 insgesamt genommenen Maße $3(0,45 \%)$ solcher Korrekturen notwendig.

Jeder Schädel wurde in drei Durchgängen (mit jeweils anderer Aufeinanderfolge von Schädeln) von jedem Autor vermessen. Als erster maß J. R., dann H. M. S., jeder an zwei Tagen, um Ermüdung auszuschalten.

Wir verwendeten beide die Schublehre von H. M. S. (Ablesegenauigkeit von $0.05 \mathrm{~mm}$ ). Dadurch war J. R. benachteiligt, da er nicht nur weniger Erfahrung im Messen von kleinen Nagerschädeln besaß, sondern noch dazu mit einem ungewohnten Gerät arbeitete. H. M. S. hatte mit demselben schon ebwa 50.000 Schädelmaße von Kleinsäugern abgenommen. J. R. übte vor dem Versuch kurz mit der Schublehre.

Die gewonnenen Werte wurden je Person und Schädelmaß mit Hilfe einer Varianzanalyse verglichen. Wir danken Herrn Doz. Dr. Alois Essl (Institut für Tierproduktoin der Universität für Bodenkultur in Wien) nicht nur für die Beratung hinsichtlich der statistischen Auswertung, sondern auch dafür, die elektronische Tischrechenmaschine (Olivetti Programm 101) seines Institut hiebei benutzen zu dürfen.

Wir berechneten die Wiederholbarkeit W, die als Korrelation zwischen den drei Messungen jedes Einzelmerkmales pro Person definiert ist, nach der Formel

$$
W=\frac{S^{2}(I)}{S^{2}(I)+S^{2}(F)}
$$

$S^{2}(I)=$ Varianzanteil, welcher auf Individualunterschiede zurückgeht

$S^{*}(F)=$ Varianzanteil, welcher auf den Meßfehler zurückgeht

Weiters wurde der relative Genauigkeitszuwachs in $\%(G Z \%, n)$ von zwei, drei oder $n$ beliebigen Messungen gegenüber nur einer berechnet.

$$
G Z \%, n=\frac{n}{1+(n-1) W}-1 \times 100
$$

In Tab. 1 wurden ferner noch Standardabweichung des Fehlers und Standardabweichung des Fehlers in Prozent des Mittelwertes angegeben.

\section{DIE AUSGEW ÄHLTEN SCHÄDELMASSE}

Beim Vermessen von Kleinsäugerschädeln mit der Schublehre kann man folgende Vorgangsweisen unterscheiden:

a) Die Meßstrecke liegt zwischen den beiden großen Schenkeln des Gerätes (die für Außenmaße verwendet werden). Die Schublehre wird so lange zusammengeschoben, bis sie beide Meßpunkte berührt. Die genauesten Maße gehören hieher, in unserem Fall: Condylobasallänge 
$(C B)$, Zygomatikbreite $(Z y g)$, Obere Zahnreihenlänge (OZR), Rostralhöhe $(R H)$. Bei der Schädelhöhe $(S H)$ sind die Meßpunkte schwer zu definieren, sodaß sich ein größerer Fehler ergibt.

b) Die beiden Meßpunkte werden mit den, einer Innenmessung dienenden, kurzen Schenkeln der Schublehre abgetastet, wobei diese auseinandergeschoben wird, bis die Meßpunkte ertastet sind.

Beispiel: Diastemalänge (Dia)

c) Einer oder beide Meßpunkte werden optisch anvisiert, Länge der $\mathrm{Na}$ salia (Nas), Länge der Foramina incisiva (FI).

\section{Tabelle 1}

Extremmaße (Min, Max) und Mittel ( $\bar{x})$ der drei Messungen je Merkmal und Person (Initialen der Autoren) sowie Standardabweichung $\left(S_{F}\right)$ des Fehlers und Standardabweichung des Fehlers in Prozent des Mittels $\left(S_{F} \%\right)$. Ferner Wiederholbarkeit $(W)$ und prozentueller Genauigkeitszuwachs von zwei $(G Z \%, 2)$ und drei $(G Z \%, 3)$ Messungen gegenüber einer Messung.

\begin{tabular}{|c|c|c|c|c|c|c|c|c|c|}
\hline & & Min & $\overline{\mathrm{x}}$ & Max & $S_{F}$ & $S_{F} \%$ & W & $G Z \%, 2$ & $G Z \%, 3$ \\
\hline$C B$ & $\begin{array}{l}\text { JR } \\
\text { HMS }\end{array}$ & $\begin{array}{l}23,8 \\
23,6\end{array}$ & $\begin{array}{l}25,42 \\
25,19\end{array}$ & $\begin{array}{l}26,6 \\
26,5\end{array}$ & $\begin{array}{l}0,088 \\
0,069\end{array}$ & $\begin{array}{l}0,347 \\
0,272\end{array}$ & $\begin{array}{r}0,985 \\
0,992\end{array}$ & $\begin{array}{l}0,739 \\
0,377\end{array}$ & $\begin{array}{l}0,989 \\
0,503\end{array}$ \\
\hline Zyg & $\begin{array}{l}\text { JR } \\
\text { HMS }\end{array}$ & $\begin{array}{l}13,1 \\
13,0\end{array}$ & $\begin{array}{l}14,04 \\
13,92\end{array}$ & $\begin{array}{l}14,7 \\
14,5\end{array}$ & $\begin{array}{l}0,053 \\
0,046\end{array}$ & $\begin{array}{l}0,376 \\
0,329\end{array}$ & $\begin{array}{l}0,987 \\
0,989\end{array}$ & $\begin{array}{l}0,671 \\
0,531\end{array}$ & $\begin{array}{l}0,897 \\
0,710\end{array}$ \\
\hline$R H$ & $\begin{array}{l}\text { JR } \\
\text { HMS }\end{array}$ & $\begin{array}{l}4,3 \\
4,2\end{array}$ & $\begin{array}{l}4,68 \\
4,55\end{array}$ & $\begin{array}{l}4,9 \\
4,9\end{array}$ & $\begin{array}{l}0,069 \\
0,037\end{array}$ & $\begin{array}{l}1,465 \\
0,822\end{array}$ & $\begin{array}{l}0,919 \\
0,973\end{array}$ & $\begin{array}{l}4,200 \\
1,370\end{array}$ & $\begin{array}{l}5,680 \\
1,835\end{array}$ \\
\hline OZR & $\begin{array}{l}\text { JR } \\
\text { HMS }\end{array}$ & $\begin{array}{l}3,80 \\
3,75\end{array}$ & $\begin{array}{l}4,13 \\
4,05\end{array}$ & $\begin{array}{l}4,45 \\
4,50\end{array}$ & $\begin{array}{l}0,060 \\
0,033\end{array}$ & $\begin{array}{l}1,452 \\
0,819\end{array}$ & $\begin{array}{l}0,882 \\
0,965\end{array}$ & $\begin{array}{l}6,265 \\
1,804\end{array}$ & $\begin{array}{l}8,532 \\
2,420\end{array}$ \\
\hline SH & $\begin{array}{l}\text { JR } \\
\text { HMS }\end{array}$ & $\begin{array}{l}9,5 \\
9,4\end{array}$ & $\begin{array}{r}10,00 \\
9,75\end{array}$ & $\begin{array}{l}10,5 \\
10,3\end{array}$ & $\begin{array}{l}0,075 \\
0,095\end{array}$ & $\begin{array}{l}0,755 \\
0,973\end{array}$ & $\begin{array}{l}0,919 \\
0,867\end{array}$ & $\begin{array}{l}4,200 \\
7,109\end{array}$ & $\begin{array}{l}5,680 \\
9,709\end{array}$ \\
\hline Dia & $\begin{array}{l}\text { JR } \\
\text { HMS }\end{array}$ & $\begin{array}{l}6,5 \\
7,0\end{array}$ & $\begin{array}{l}7,36 \\
7,55\end{array}$ & $\begin{array}{l}8,0 \\
8,2\end{array}$ & $\begin{array}{l}0,178 \\
0,059\end{array}$ & $\begin{array}{l}2,414 \\
0,783\end{array}$ & $\begin{array}{l}0,791 \\
0,970\end{array}$ & $\begin{array}{r}11,678 \\
1,535\end{array}$ & $\begin{array}{r}16,201 \\
2,057\end{array}$ \\
\hline Nas & $\begin{array}{l}\text { JR } \\
\text { HMS }\end{array}$ & $\begin{array}{l}9,8 \\
9,8\end{array}$ & $\begin{array}{l}10,35 \\
10,31\end{array}$ & $\begin{array}{l}10,7 \\
10,9\end{array}$ & $\begin{array}{l}0,109 \\
0,056\end{array}$ & $\begin{array}{l}1,053 \\
0,539\end{array}$ & $\begin{array}{l}0,887 \\
0,963\end{array}$ & $\begin{array}{l}6,002 \\
1,907\end{array}$ & $\begin{array}{l}8,167 \\
2,559\end{array}$ \\
\hline$F I$ & $\begin{array}{l}\text { JR } \\
\text { HIMS }\end{array}$ & $\begin{array}{l}5,1 \\
4,9\end{array}$ & $\begin{array}{l}5,39 \\
5,35\end{array}$ & $\begin{array}{l}5,6 \\
5,7\end{array}$ & $\begin{array}{l}0,048 \\
0,090\end{array}$ & $\begin{array}{l}0,889 \\
1,681\end{array}$ & $\begin{array}{c}0,902 \\
0,823\end{array}$ & $\begin{array}{l}5,145 \\
9,694\end{array}$ & $\begin{array}{r}6,980 \\
13,357\end{array}$ \\
\hline
\end{tabular}

Alle Maße wurden wie in St e in er (1968) beschrieben abgenommen. Die Werte wurden auf $0,05 \mathrm{~mm}$ genau abgelesen, jedoch sofort auf $0,1 \mathrm{~mm}$ gerundet; nur die $O Z R$ wurde auf $0,05 \mathrm{~mm}$ genau notiert.

\section{DISKUSSION DER ERGEBNISSE}

4.1. Condy lobas allänge. Die Wiederholbarkeit der einzelnen Messungen dieses Merkmales ist erwartungsgemäß hervorragend. Beim Geübten ist der Genauigkeitszuwachs von drei Messungen gegenüber nur einer bloß $0.5 \%$ (Tab. 1). Absolute Größe und Ablesegenauigkeit stehen in einem günstigen Verhältnis. 
Bei dieser Meßgenauigkeit ist es sicher, daß der bei den einzelnen Schädeln durchgehende Unterschied zwischen den Personen (im Mittel liegen die Werte von J. R. 0,23 mm höher) auf verschiedene Maßtechniken zurückgeht. Bei einem nochmaligen Vergleich nach Ende des Versuches zeigte er sich, daß J. R. nach Zusammenschieben des Schenkel der Schublehre den Schädel herausnahm und dann ablas, H. M. S. den zu messenden Schädel zart einklemmte, ihn zur Probe mit dem Gerät hochhob und dann ablas.

4.2. Z y g o matikbreite. Die Wiederholbarkeit ist auch hier ausgezeichnet, der individuelle Meßfehler dürfte ähnlich wie bei der $C B$ bedingt sein. Für die Charakterisierung erwachsener Apodemus hervorragend geeignet; bei jüngeren Tieren, deren skelettierte Schädel noch keine konvex ausgeformten Jugalia besitzen, sind jedoch wesentlich schlechtere Ergebnisse zu erwarten.

4.3. Rostralhöh e. Hiemit wurde ein absolut kleines $\mathrm{Ma}$ gewählt. Es ist zur Charakterisierung von Schädeln sicher brauchbarer als man meist annimmt und liegt im oft allein intakt gebliebenen Rostrum. Leider kann es an Gewöllresten nicht gemessen werden!

Bezogen auf die ungünstige Relation von Größe und Ablesegenauigkeit (Rundungsfehler!) erscheint die Wiederholbarkeit gut. Die individuellen Unterschiede sind in der Erfahrung begründet: die Stelle der geringsten Höhe des Rostrums kann mit der Schublehre gut ertastet werden.

4.4. Obere $\mathrm{Zahnreihenlänge.} \mathrm{Dieses} \mathrm{wertvolle} \mathrm{Zahnmaß} \mathrm{kann}$ bei fast allen Tieren an der Zahnkrone vergleichbar ertastet werden: nur ganz junge Exemplare und senile machen eine Ausnahme (S t e :ner, 1968; N i eth a m m e r, 1969). Bezogen auf die geringe absolute Größe kann die $O Z R$ präzise vermessen werden (Tab. 1). Es darf nicht vergessen werden, daß durch den Verzicht auf Rundung bei diesem Merkmal die Wiederholbarkeit bei gleicher Meßgenauigkeit geringer sein muß, ist doch die Chance beim Messen in die halb so großen Meßklassen ,richtig” zu treffen, weniger groß.

4.5. S c hä delhöh e. Die Schwierigkeit des genauen Definierens der Meßpunkte ist wohl schuld, wenn bei keinem von uns die Zunahme der Genauigkeit von den drei Messungen gegenüber einer unter $5^{\mathbf{0}} \%$ lag. Hieran gemessen ist die 2,5 fach größere $C B$ zehnfach genauer! J. R. maß von den Bullae auditorii von einer gedachten Unterlage, auf der der Schädel liegen würde, senkrecht nach oben, H. M. S. wählte dic Oberseite der Interparietalia als zweiten Meßpunkt. Die Zahlen von Tab. 1 beweisen, daß die Methode von J. R. exakter ist.

4.6. Diastema länge. Das Diastem ist bei zerschlagenen Schädeln oft das größte bestimmbare Maß; dies gilt auch für gut erhaltene Gewöllschädel. Gemessen wird alveolär. 
Es werden die kurzen Schenkel der Schublehre verwendet (vgl. Kapitel 2), die in zwei Ebenen aneinander vorbeigleiten. Deshalb ist es wichtig, daß immer die gleiche Körperseite vermessen wird. Die Meßstrecke liegt nicht parallel zur Körper-Mittelebene. Bei entsprechender Übung ist die Wiederholbarkeit von Diastema-Messungen gut. Vergleiche zwischen verschiedenen Autoren sollen hingegen vorsichtig durchgeführt werden.

4.7. Länge der Nasalia. Die Genauigkeit der Messungen hängt in diesem Fall davon ab, wie präzise der Hinterrand der Sutura nasofrontalis mit dem einen Schenkel der Schublehre zur optischen Deckung gebracht werden kann. (Bei gebleichten Schädeln ist dies viel schwieriger als bei unseren ungebleichten!). Unsere Erwartungen waren in Bezug auf dieses Maß nicht sehr hochgespannt. Wir waren von den günstigen Wiederholbarkeitswerten und vor allem vom geringen Unterschied zwischen den Mittelwerten der beiden Meßpersonen verblüfft. (Tab. 1).

4.8. Länge der Foramina incisiva. Durch den Formenunterschied hinsichtlich dieses Merkmales zwischen den drei Arten von Sylvaemus ( $\mathrm{S}$ t e in er, 1968; N i e tha m mer, 1969) besitzt es große Bedeutung.

Wir hatten vor dem Versuch nicht besprochen, ob wir dieses Maß unter dem Stereomikroskop messen sollten. J. R. verwendete ein solches bei 10 -facher Vergrößerung um über die Schenkel der Schublehre zu visieren, H. M. S. nicht. Dadurch ist wohl die Vergleichbarkeit geschmälert, aber dafür gezeigt, wie wichtig bei optisch anzuvisierenden Meßpunkten ein optisches Hilfsmittel ist. Die Meßgenauigkeit dürfte bei Úbung weiter zu steigern sein (Tab. 1).

\section{SCHLUSSFOLGERUNGEN}

Schädelmaße, deren exakt definierbaren Meßpunkte zwischen den Schenkeln der Schublehre ertastet werden können, sind im allgemeinen am exaktesten zu messen $(C B, Z y g, R H$ und $O Z R)$. Der Genauigkeitszuwachs von drei Messungen je Schädel gegenüber nur einer, dürfte bei größeren Maßen $(C B, Z y g)$ wohl immer unter $1 \%$ zu drücken sein, bei kleineren $(R H, O Z R) 2 \%$ wenig überschreiten. Ein - besser eingearbeiteter Untersucher wird die Schublehre wiederholbar knapper an die Meßpunkte heranführen und geringere Werte erhalten.

Auch die Schädelhöhe kann man zwischen den Meßschenkeln ertasten. Bei Vergleichen zwischen Personen ist aber wegen der Schwierigkeit der genauen Definition der Meßstrecke ein großer Fehler zu erwarten.

Beim Messen der Diastemalänge liegen die kurzen Schenkel der 
Schublehre zwischen den Meßpunkten. Bei großer Vorsicht kann auch hier sehr exakt gemessen werden. In diesem Falle wird der Geübte bei sonst vergleichbaren Bedingungen die größere Distanz ertasten. Verschieaen dicke Meßschenkel verschiedener Schublehren dürften einen großen Einfluß haben, sodaß Vergleiche zwischen Autoren nicht sehr präzise möglich sein dürften.

Es wird empfohlen, bei mechanisch ertastbaren Meßstrecken den zart zwischen den Schenkeln der Schublehre eingeklemmten Schädel mit

\section{Tabelle 2}

Anzahl der Differenzen zwischen den Extremwerten innerhalb der drei Messungen je Schädel, Merkmal und Person in den einzelnen mit $0,1 \mathrm{~mm}$ abgestuften Differenzklassen.

\begin{tabular}{|c|c|c|c|c|c|c|c|c|c|c|}
\hline & & 0,0 & 0,1 & 0,2 & 0,3 & 0,4 & 0,5 & 0,6 & 0,7 & $\mathrm{n}$ \\
\hline \multirow[t]{2}{*}{$C B$} & JR & 2 & 6 & 5 & 1 & & & & & 14 \\
\hline & HMS & 7 & 4 & 2 & 1 & & & & & 14 \\
\hline \multirow[t]{2}{*}{ Zyg } & JR & 2 & 12 & & & & & & & 14 \\
\hline & $\begin{array}{l}\text { HMS } \\
\text { JR }\end{array}$ & $\begin{array}{l}9 \\
5\end{array}$ & $\begin{array}{l}3 \\
7\end{array}$ & 2 & & & & & & 14 \\
\hline$R H$ & HMS & 8 & 6 & & 1 & & & & & $\begin{array}{l}14 \\
14\end{array}$ \\
\hline \multirow[t]{2}{*}{$S H$} & JR & & 10 & 4 & & & & & & 14 \\
\hline & HMS & 1 & 7 & 5 & & 1 & & & & 14 \\
\hline Dia & $\begin{array}{l}\text { JR } \\
\text { HMS }\end{array}$ & $\begin{array}{l}1 \\
5\end{array}$ & $\begin{array}{l}1 \\
8\end{array}$ & 7 & 1 & 3 & 1 & & 1 & $\begin{array}{l}14 \\
14\end{array}$ \\
\hline \multirow[t]{2}{*}{ Nas } & JR & 2 & 4 & 5 & 2 & 1 & & & & 14 \\
\hline & HMS & 4 & 9 & 1 & & & & & & 14 \\
\hline \multirow[t]{2}{*}{$F I$} & JR & 7 & 6 & 1 & & & & & & 14 \\
\hline & HMS & 1 & 7 & 4 & & 2 & & & & 14 \\
\hline
\end{tabular}

Tabelle 3

Anzahl der Differenzen zwischen den Extremwerten innerhalb der drei Messungen je Schädel und Person bei der Oberen Zahnreihenlänge in den mit $0,05 \mathrm{~mm}$ abgestuften Differenzklassen.

\begin{tabular}{lllllllll}
\hline & & 0,0 & 0,05 & 0,10 & 0,15 & 0,20 & 0,25 & n \\
\hline OZR & JR & 2 & 8 & 1 & 1 & & 2 & 14 \\
& HMS & 3 & 8 & 3 & & & & 14 \\
\hline
\end{tabular}

dieser hochzuheben um sich von der exakten Erfassung der Meßpunkte zu überzeugen.

Bei optisch anvisierten Meßpunkten, in unserem Fall Nas und FI, wird die Benutzung eines Stereomikroskop dringend empfohlen.

Wir wollten bei unserer Untersuchung nicht nur Meßmethoden vergleichen und die Empfehlbarkeit einzelner Maße prüfen, sondern wir wollten auch einen Vorschlag ausarbeiten, wie jeder Untersucher ohne komplizierte Berechnungen seine Meßgenauigkeit abschätzen kann. Wir 
haben deshalb die Differenz der Extremwerte der drei Messungen je Schädel, Merkmal und Person in den Tab. 2 und 3 dargestellt. Jeder kann bei Mithilfe einer Person seine eigene Technik prüfen, indem er die Ergebnisse von Meßversuchen in ähnliche Tabellen einträgt. Sodann kann er aus dem Vergleich seiner eigenen Werte mit denen unserer Tab. 1-3 grob abschätzen, wieweit seine Methode genau ist.

Es wird vermutet, daß gröbere Ablesefehler (z.B. um ganze Millimeter) in Serien häufiger Extremwerte vortäuschen als man annimmt, weswegen diese überprüft werden sollten.

Wiederholungen von Messungen sind sonst im allgemeinen wohl nur dort rationell, wo der Beschreibung eines oder weniger Exemplare großes Gewicht zukommt.

\section{LITERATUR}

1. Haitlinger R. \& Ruprecht A. L., 1967: The taxonomic value of teeth measurements in the subgenus Sylvaemus Ognev \& Vorobiev, 1923. Acta theriol., 12: $180-187$.

2. Niethammer J., 1969: Zur Frage der Introgression bei den Waldmäusen Apodemus sylvaticus und A. flavicollis (Mammalia, Rodentia). Z. zool. Syst. Evolutionsforsch., 7: 77-127.

3. Ste in er H. M., 1968: Untersuchungen über die Variabilität und Bionomie der Gattung Apodemus (Muridae, Mammalia) der Donau-Auen von Stockerau (Niederösterreich). Z. wiss. Zool., 177: 1-96.

Accepted, June 18, 1976.

Hans M. STEINER i Jan RACZYŃSKI

POWTARZALNOSC POMIAROW I INDYWIDUALNY BEĄD POMIAROWY W BADANIACH KRANIOMETRYCZNYCH NA PRZYKEADZIE APODEMUS

\section{Streszczenie}

Na serii 14 czaszek Apodemus flavicollis (M elchior, 1834) dwóch eksperym€ntatorów wykonało niezależnie po 8 indywidualnych pomiarów czaszki w 3 powtórzeniach. Z użyciem analizy wariancji wyliczono dla obydwu mierzących powtarzalność $(W)$ i procentowy wzrost dokładności $(G Z \%)$ indywidualnych pomiarów w kolejnych powtórzeniach (Tabela 1) oraz zestawiono odchylenia wartości poszczególnych pomiarów (Tabela 2). Największą dokładność ,zdejmowania” pomiaru stwierdzono dla długości kondylobazalnej (CB) i szerokości zygomatycznej $(Z y g)$; wysoką dla wysokości rostrum $(R H)$, długości górnego szeregu zębów (OZR), dług. diastemy (Dia) i długości kości nosowych (Nas). Mierzalność wysokości puszki mózgowej $(\mathrm{SH})$ i długości Foramina incisiva (FI) była niezadowalająca. Autorzy dyskutują wpływ punktów pomiarowych i techniki mierzenia na dokładność pomiaru i zalecają użycie prostej metody testowania indywidualnego błędu (por. Tabela 3) oraz powtarzanie mierzenia w przypadku opracowywania nielicznych kolekcji czaszek. 